\title{
Heavy-light decay constants on the lattice
}

UKQCD Collaboration

Presented by C.M. Maynard ${ }^{\mathrm{a}}$

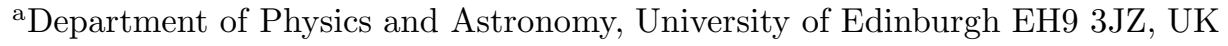

Edinburgh-2001/13

We present results for the leptonic decays of heavy-light mesons in the quenched approximation and a preliminary result for $f_{D_{s}}$ with dynamical fermions for $N_{f}=2$. We examine the systematic effects of extrapolating the decay constants from the charm to the bottom quark mass, and find this extrapolation to be under control. We present a preliminary result for $f_{D_{s}}^{N_{f}=2}$ and compare to $f_{D_{s}}^{N_{f}=0}$ matched to have the same lattice spacing set by the Sommer scale, $r_{0}$. We find no significant difference.

\section{INTRODUCTION}

The decay constants of the $\mathrm{B}$ and $\mathrm{D}$ mesons are important in phenomenology. The combination $f_{B} \sqrt{B_{B}}, f_{B_{s}} \sqrt{B_{B_{s}}}$, and their ratio, play a crucial role in extracting CKM matrix elements from experimental data. The B-factory experiments BaBar and Belle will measure $B^{0}-\bar{B}^{0}$ mixing quantities, but measuring $f_{B}$ will be very difficult.

On the lattice, $f_{B}$ is a mature calculation, and less extensive than $B_{B}$, so we can test the systematics of a heavy-light lattice calculation. The "world average" for $f_{B}$ [1] in the quenched approximation is $f_{B}=175(20) \mathrm{MeV}$ and with dynamical fermions (DF), $f_{B}^{N_{f}=2}=200(30) \mathrm{MeV}$ (see also [2]). This suggests a strong dependence on sea quark effects. However, it is not clear how to disentangle different systematic effects.

\section{QUENCHED DECAY CONSTANTS}

These results have been partially reported in [3,4]. We use the non-perturbatively (NP) $\mathcal{O}(a)$ improved Wilson quark action at two values of the coupling, $\beta=6.2$ and $\beta=6.0$. The light quarks have masses around strange, and the heavy quarks around charm. In the NP improvement scheme, the axial current is given by

$A_{\mu}^{\mathrm{R}}=Z_{A}\left(1+b_{A} a m_{q}\right)\left(A_{\mu}+a c_{A} \partial_{\mu} P_{s}\right)$

We use non-perturbative determinations of the coefficients $Z_{A}, b_{A}$ and $c_{A}$ by Bhattacharya et al. [5]. In [3] we show that the value of the decay constant depends strongly on the value of the mixing coefficient $c_{A}$. These coefficients are only defined up to $\mathcal{O}(a)$, e.g. compare the ALPHA [6] and Bhattacharya et al. values of $c_{A}$ at $\beta=6.0$.
$c_{A}^{\mathrm{ALPHA}}=-0.083(5)$ and $c_{A}^{\mathrm{Bhatt}}=-0.037(4)(7)$. However, observables computed with these coefficients have the same continuum limit, provided each set of coefficients is determined consistently.

We use continuum Heavy Quark Symmetry (HQS) to motivate the form of the extrapolation to the $b$ quark mass;

$\Phi(M)=\Theta(M) f \sqrt{M}=\gamma\left(1+\frac{\delta}{M}+\frac{\eta}{M^{2}}\right)$

where $M$ is the pseudoscalar meson mass, $f$ the pseudoscalar decay constant defined by

$\left\langle 0\left|A_{\mu}\right| P_{s}(\vec{p})\right\rangle=i f p_{\mu}$

and $\Theta$ is the logarithmic correction from QCD.

This extrapolation is shown in figure 1. We have also computed the value of $\Phi$ in the static limit, but have not included the improvement term. We note that $c_{A}$ is negative, which will reduce the value of $\Phi$. We do not interpolate from charm, but use the static point as a check in the extrapolation.

Also shown in figure 1 is a change of normalisation of the axial current labelled $\mathrm{CB}$, as suggested by Bernard [1],

$\left(A_{0}^{R}\right)_{\mathrm{CB}}=Z_{A}\left(1+b_{A} a m_{q}+2 a c_{A} \mathcal{R}\right)^{1 / 2} A_{0}$

where $\mathcal{R}=\partial_{0}\langle 0|P| P\rangle / m_{q}\left\langle 0\left|A_{0}\right| P\right\rangle$ This differs from the normalisation in equation (11) at $\mathcal{O}\left(a^{2}\right)$, but has a finite static limit. This is plotted against $M_{2}$, the kinetic mass, $M_{2}^{-1}=$ $\partial^{2} E /\left.\partial p_{i}^{2}\right|_{\vec{p}=0}$ There is a small difference between our standard extrapolation and that labelled CB.

To estimate the effect of mass dependent lattice artefacts directly from the data, we add terms to equation (2) that model the lattice artefacts,

$\Phi^{\prime}(M, a)=\Phi(M)+\gamma\left(\varepsilon(a M)^{2}+\zeta(a M)^{3}\right)$ 
2

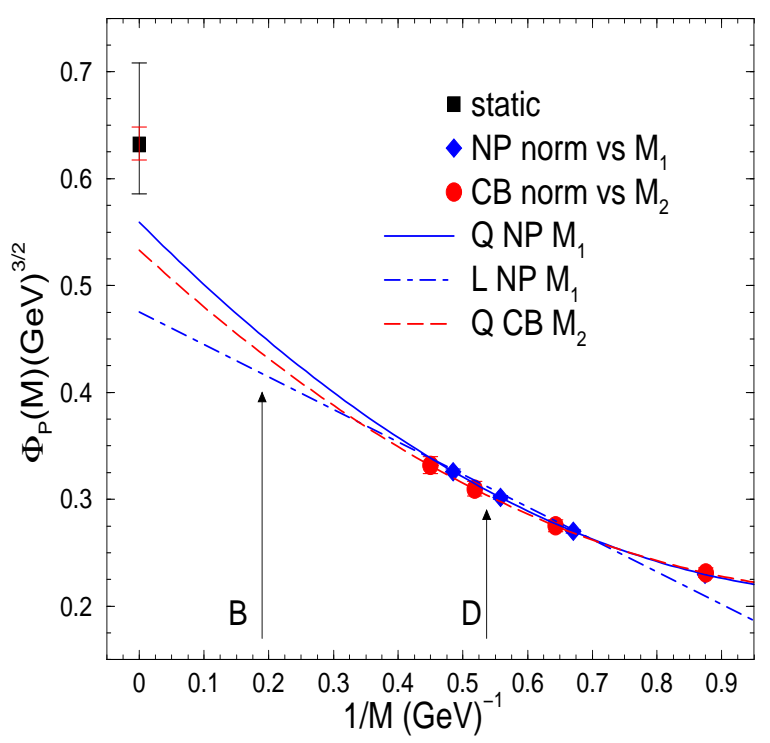

Figure 1. HQS extrapolation of $\Phi$ at $\beta=6.0$

We then fit to both lattice spacings simultaneously. Shown in figure 2 is the data at both values of $\beta$. The fit parameters $\varepsilon$ and $\zeta$ are small and consistent with zero. Line $\mathrm{A}(\mathrm{B})$ shows the function $\Phi^{\prime}(M, a)$ with $a$ at $\beta=6.2(6.0)$. These show that lattice artefacts indeed do blow up if extrapolated. However, the lines labelled " $\beta=6.2(6.0)$ only" and "quasi-continuum" (QC) $\left(\Phi^{\prime}(M, 0)\right)$, lie close together. Moreover $f_{B}^{\mathrm{QC}}=186(12) \mathrm{MeV}$, is close to the values of $f_{B}$ at fixed lattice spacing, albeit with larger errors, which implies mass dependent lattice artefacts are not spoiling the extrapolation.

We estimate the systematic uncertainties from the quantity used to fix $a,\left(r_{0}, m_{\rho}\right)$, linear vs quadratic HQS extrapolation, quasi-continuum extrapolation and the difference between different lattice spacings. We observe good scaling between different lattice spacings. The values for the quenched decay constants are as follows, with the scale set by $f_{\pi}$. The errors are statistical and systematic;

$$
\begin{array}{rlrl}
f_{B} & =195(6)_{-23}^{+24} \mathrm{MeV} & f_{D}=206(4)_{-10}^{+17} \mathrm{MeV} \\
f_{B_{S}}=220(6)_{-28}^{+23} \mathrm{MeV} & f_{D_{S}}=229(3)_{-12}^{+23} \mathrm{MeV} \\
\frac{f_{B_{s}}}{f_{B}}=1.13(1)_{-5}^{+1} & \frac{f_{D_{s}}}{f_{D}}=1.11(1)_{-3}^{+1} \\
f_{K}=150(3)_{-8}^{+12} \mathrm{MeV} & &
\end{array}
$$

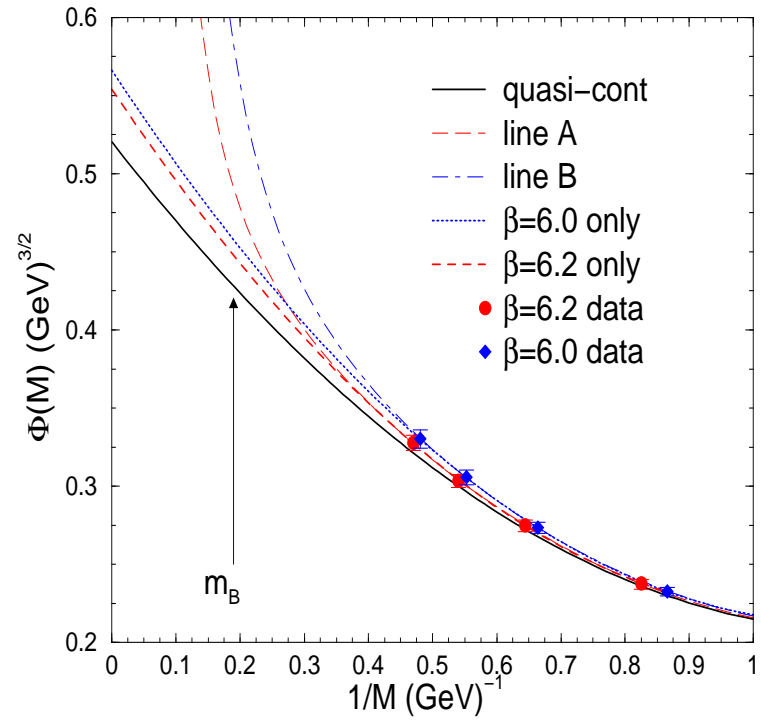

Figure 2. Quasi-Continuum extrapolation of $\Phi$

\section{DECAY CONSTANTS WITH $N_{f}=2$}

The UKQCD collaboration has a set of matched ensembles [7], where the lattice spacing (as measured by $r_{0}$ ) is fixed to be the same, as $\beta$ and $m_{q}^{\text {sea }}$ are varied, including a quenched set. These ensembles are NP $\mathcal{O}(a)$ improved. Measuring the decay constants on these ensembles will enable us to study the effect of unquenching whilst keeping everything else fixed. Furthermore, we intend to study the systematics of different heavy quark methods by comparing NRQCD, FNAL formalism and charm plus extrapolation directly on the same data sets. In particular it is interesting to see if the ratio $f_{B}^{N_{f}=2} / f_{B}^{N_{f}=0}$ is the same for each method.

We present a preliminary result for $f_{D_{s}}$ at fixed lattice spacing, comparing the $N_{f}=2$ value with the quenched value. The simulation details are shown in Table 1 .

Table 1

Simulation details

\begin{tabular}{ccc}
\hline & $N_{f}=2$ & $N_{f}=0$ \\
\hline$\beta, \kappa^{\text {sea }}$ & $5.2,0.1350$ & $5.93,0$ \\
$m_{\pi} / m_{\rho}$ & $0.70(1)$ & 1 \\
$c f g s$ & 202 & 117 \\
$a^{-1}$, volume & $0.1 \mathrm{fm}, 16^{3} \times 32$ \\
valence light & \multicolumn{2}{c}{$3 \sim$ strange } \\
valence heavy & \multicolumn{2}{c}{$4 \sim$ charm } \\
$c_{A}$ BPT & -0.0164 & -0.0132 \\
$c_{A} \mathrm{NP}$ & $-0.04(2)$ & $-0.032(14)$ \\
\hline
\end{tabular}


The values of the improvement coefficients have been determined to one-loop in perturbation theory [8]. At one-loop these are independent of sea quark effects. We evaluate these in "boosted" perturbation theory. The interpolation to charm is shown in figure 3. We see no significant difference.

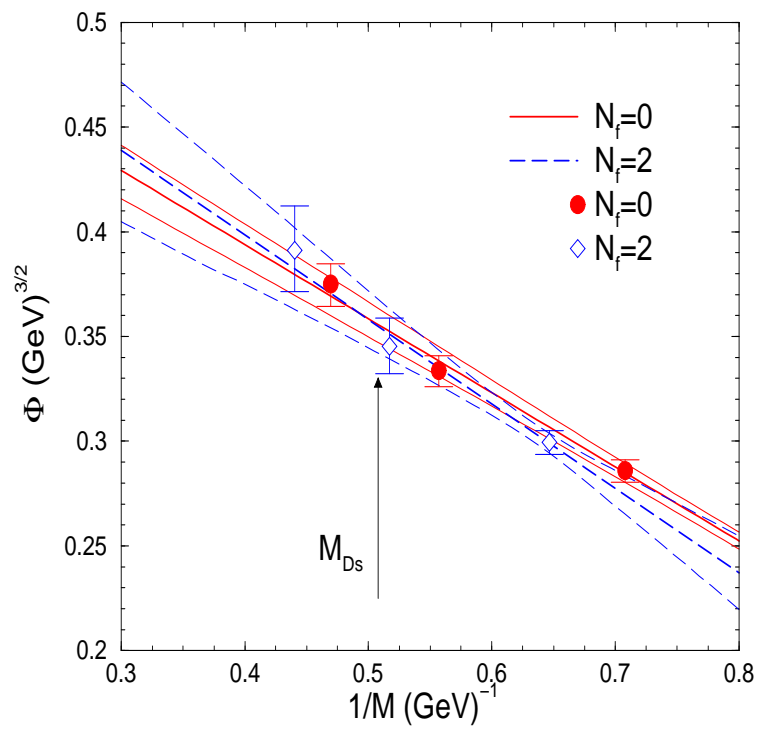

Figure 3. Comparison between DF and quenched data

There is a preliminary NP determination [9]. Table 2 shows the dependence of $f_{D_{s}}$ on the value of $c_{A}$. The entry $P_{S}$ shows the value of the pseudoscalar density contribution.

Table 2

$f_{D_{s}}$ in $\mathrm{MeV}$ and its dependence on $c_{A}$.

\begin{tabular}{lcc}
\hline$c_{A}$ & $N_{f}=2$ & $N_{f}=0$ \\
\hline $\mathrm{BPT}$ & $253(9)$ & $253(6)$ \\
$\mathrm{NP}$ & $237(9)$ & $241(6)$ \\
0 & $264(9)$ & $262(6)$ \\
$P_{S}$ & $693(24)$ & $629(14)$ \\
\hline
\end{tabular}

Other than a small difference in the improvement term there is no difference. One reason may be the sea quarks we have used are still rather heavy. The Sommer scale $r_{0}$ is a short distance quantity and the decay constant, being proportional to the wavefunction at the origin is also a short distance quantity. We have fixed $a$ with a short distance quantity, thus the short distance physics will be similar for both quenched and unquenched. It is not surprising that we observe no difference. The MILC collaboration [10] see a similar pattern. Little difference when the scale is set by $r_{1}$ (related to $\left.r_{0}\right)$ and significant $m_{q}^{\text {sea }}$ dependence when the scale is set by $m_{\rho}$. The decay constants are dimensionfull quantities, so they are are sensitive to the scale setting ambiguity in the quenched approximation. The change in fDs from unquenching depends very strongly on how we choose to set the scale.

We acknowledge the following grants; EPSRC GR/K41663, PPARC PPA/G/S/1999/00022 and PPA/GS/1997/00655 and EU HPRN-CT-200000145 Hadrons/LatticeQCD. CMM acknowleges PPA/P/S/1998/00255 and thanks C. Bernard, P. Boyle, S. Collins, J.Hein, C.McNeile, and S. Ryan for discussions and advice.

\section{REFERENCES}

1. C. Bernard, Nucl. Phys. B (Proc. Suppl.) 94, 159 (2001).

2. S. Ryan, these proceedings.

3. UKQCD Collaboration, K.C. Bowler et al., hep-lat/0007020.

4. UKQCD Collaboration, C.M. Maynard, Nucl. Phys. B (Proc. Suppl.) 94, 367 (2001).

5. T. Bhattacharya, R. Gupta, W. Lee, S. Sharpe, Phys. Rev. D 62, 074505 (2001).

6. M. Lüscher, S. Sint, R. Sommer, P. Weisz, H. Wittig and U. Wolff, Nucl. Phys. B (Proc. Suppl.) 53, 905 (1997).

7. UKQCD Collaboration, A.C. Irving et al., Phys. Rev. D 58, 114504 (1998).

8. S. Sint and P. Weisz, Nucl. Phys. B 502, 251 (1997).

9. UKQCD collaboration, S. Collins et al., these proceedings.

10. MILC collaboration, C. Bernard et al., these proceedings. 